\title{
USO Y ABUSO DEL ESTUDIO RADIOLOGICO DE ESOFAGO, ESTOMAGO Y DUODENO EN PACIENTES PEDIATRICOS: NECESIDAD DE UNA ADECUADA NORMATIVA Y DE UNA ESTANDARIZACION DEL EXAMEN
}

\author{
Drs. Cristián García $B^{(1,2)}$, Luis Meneses $Q^{(1)}$, Ernesto Guiraldes $C^{(2)}$, Dimitri Parra $R^{(1)}$, José $D$. Arce $V^{(3)}$, \\ Giancarlo Schiapaccasse $F^{(1)}$, Veruska de Luccas ${ }^{(1)}$, Alejandro Bustos $A^{(1)}$, Rodrigo Parra $R^{(1)}$, Víctor Díaz $B^{(1)}$.
}

\section{Departamento de Radiología. Pontificia Universidad Católica de Chile. \\ 2. Departamento de Pediatría. Pontificia Universidad Católica de Chile. \\ 3. Servicio de Radiología. Clínica Santa María Santiago.}

\begin{abstract}
Introduction: The role of the upper gastrointestinal radiography study (UGIR) in the evaluation of children with vomiting, has not been well defined in our country, specially regarding its role in the diagnosis of gastroesophageal reflux (GER). GER is in most cases a physiologic condition, manifested by vomiting and/or regurgitation in infants. The American Academy of Pediatrics has insisted on this point, considering the UGIR as a useful study in order to rule out an anatomical cause of vomiting, but in most cases, the clinical history and physical examination are enough to make the diagnosis. Radiologists are performing examinations, in most cases unnecessary and the results are not interpreted appropriately. In a great number of patients, the UGIR is done in order to obtain a maternal license, based on our current law. Patients and Methods: A total of 190 children in whom an UGIR was performed in our Hospital, were included in a prospective study, from April 1, to September 30, 2004. In every case, many parameters were consigned, including clinical history, the reason for the study, etc. Results: This study revealed that most children referred for UGIR study, were infants with vomiting and/or regurgitation without a real indication for the study. In 46 patients (24\%), the mother was already under maternal license and in 39 (85\%) of these patients, the study was asked for because of vomiting and median age was 4 months. In those infants in whom the mothers did not have license
\end{abstract}

García $\boldsymbol{C}$, y cols. Uso y abuso del estudio radiológico de esófago, estómago y duodeno en pacientes pediátricos: Necesidad de una adecuada normativa y de una estandarización del examen. Rev Chil Radiol 2005; 11: 23-29.

Correspondencia: Dr. Cristián García B.

Departamento de Radiología. Facultad de Medicina. Pontificia Universidad Católica.

Marcoleta 367. F: 3543704•cgarcia@med.puc.cl
(114 mothers), median age was 3 months. Thirty (26\%) of these 114 mothers came to our Hospital in order to obtain a medical license based on the results of the UGIR study. GER was doccumented in 81 patients and 3 had a swallowing disorder. In none of them, an anatomical condition was detected, as the cause of vomiting. Conclusions: Our results confirm that the use of UGIR in children is totally confusing and misunderstood in our country, and in most cases it has been used in order to obtain a medical license for the mothers. We propose either changing the current law in order to extend the maternal license for all the mothers or try to find another diagnostic method. We also propose a standard protocol for the UGIR examination.

Key words: Gastroesophageal reflux, Upper gastrointestinal radiography.

Resumen. Introducción: El rol de la radiografía de esófago, estómago y duodeno (RxEED) en el estudio del niño con vómitos y en especial, en la pesquisa del reflujo gastroesofágico (RGE), en nuestro medio, es confuso. El RGE es una condición generalmente fisiológica que se manifiesta como regurgitación habitual en el lactante menor. La academia Americana de Pediatría considera la RxEED como un procedimiento útil para la evaluación de anormalidades anatómicas y reitera que en los lactantes con vómitos y regurgitación, la historia clínica y el examen físico son suficientes para formular diagnóstico, reconocer complicaciones e iniciar tratamiento. Los radiólogos estamos efectuando exámenes muchas veces innecesarios y molestos para los niños, cuyos resultados no son adecuadamente interpretados, ya sea por desconocimiento de los médicos solicitantes o por una errada normativa legal en cuanto a la extensión de licencia maternal. Se debe agregar que estamos haciendo uso inadecuado de radiación ionizante. Objetivos: Este trabajo persigue analizar 
la confusión sobre la utilidad e indicaciones de la RxEED en el estudio del niño con vómitos o regurgitación, la inadecuada indicación médica de este y la errada normativa vigente que obliga a los niños a ser sometidos a este estudio para extender una licencia postnatal de las madres. Se propone además, un intento para estandarizar la técnica de examen y establecer un consenso en cuanto a sus indicaciones e interpretación. Pacientes y Método: En forma prospectiva, se incluyó en este estudio a 190 niños, menores de 1 año, estudiados en forma consecutiva con RxEED en nuestro Hospital Clínico, desde el $1^{\circ}$ de Abril hasta el 30 de Septiembre del 2004. En cada caso se consignó numerosos antecedentes, incluyendo la técnica del examen, el cuadro clínico, el motivo del examen y un cuestionario a los padres.

Resultados: Este estudio reveló que la mayor parte de los niños referidos para RxEED, eran lactantes que solamente presentaban regurgitación y/o vómitos, sin complicaciones de ninguna especie y en la gran mayoría no existía una indicación médica real. Un $79 \%$ de los pacientes fue referido por vómitos o regurgitación y en un $6 \%$ no se especificaron causas. Sólo en un $4 \%$ se sospechó un trastorno de la deglución, que podría justificar el examen.

En 46 casos (24\%), la madre estaba ya con licencia debido al RGE de su hijo al momento del examen y en 39 de ellas (85\%) el examen se solicitó por vómitos o regurgitación, con una mediana de edad de cuatro meses. Los hijos de las 114 madres que no estaban con licencia fueron referidos al estudio por vómitos o regurgitación en un $62 \%$ de los casos y la mediana de edad fue de tres meses. Treinta de estas 114 madres (26\%) concurrieron al examen para obtener una probable concesión de licencia para su trabajo, en caso que se documentara RGE en sus hijos. En 81 pacientes hubo RGE en el examen y en tres se comprobó una alteración en la deglución. En ningún paciente se documentó anormalidad anatómica del esófago, del estómago, ni alteraciones evidentes del vaciamiento gástrico. Conclusiones: Nuestros resultados comprueban que el uso de la RxEED en el niño, ha sido notablemente desvirtuado en nuestro medio, tanto desde el punto de vista médico como social, siendo solicitado sin causas médicas justificadas en la inmensa mayoría de los casos y su indicación solo sirve de pretexto para documentar una condición fisiológica con el fin de conceder licencias de trabajo maternales.

La normativa vigente debería se modificada para extender el período de licencia maternal postnatal a todas las madres sin excepción o bien buscar otras herramienta que permita reconocer los casos que realmente requieren una extensión de ella, y liberar a los radiólogos de estar involucrados en una práctica éticamente discutible. Es necesario además estandarizar la técnica de la RxEED, en nuestro medio, para lo cual se hace una proposición.

Palabras claves: Radiografía de esófago, estómago y duodeno. Reflujo gastroesofágico,

\section{Introducción}

El reflujo gastroesofágico (RGE), se define como el paso retrógrado del contenido gástrico al esófago, constituyendo en el lactante un evento fisiológico que disminuye con la edad ${ }^{(1,2)}$. Su importancia clínica es la potencial asociación con otras patologías como el retardo ponderal, patología respiratoria o eventualmente episodios agudos amenazante de la vida (ALTE) ${ }^{(3)}$.

La Academia Americana de Pediatría ha respaldado una guía de práctica clínica destinada al manejo de pacientes con $R G E^{(4)}$. En ella se reitera que en los lactantes con vómitos y regurgitación, la historia clínica y el examen físico son suficientes para formular un diagnóstico, reconocer complicaciones e iniciar un tratamiento y generalmente no se requiere de ningún tipo de exámenes para confirmar el diagnóstico(4). Distinto es el caso de aquellos pacientes en que existe sospecha clínica de RGE, una patología asociada y no se obtiene respuesta al tratamiento médico indicado, en ellos el estudio de imágenes es necesario para descartar una causa anatómica. Es nuestra impresión que esto no ha sido bien entendido por los médicos de nuestro medio.

Los pacientes con retardo del desarrollo psicomotor o daño neurológico constituyen un grupo diferente, ya que pueden tener un importante RGE y sufrir además con mayor frecuencia complicaciones secundarias a éste ${ }^{(1)}$. Ellos requieren la certificación del diagnóstico y un tratamiento más agresivo, algunas veces quirúrgico.

En la actualidad, el examen con mayor rendimiento en el diagnóstico de RGE es la $\mathrm{pH}$ metría de 24 horas $^{(3)}$ que permite conocer además del número de episodios ocurridos en un período de 24 horas, el tiempo de duración de cada uno de ellos y el tiempo total de pH ácido en el esófago. Sin embargo, no evalúa el volumen del material refluido y su rendimiento disminuye cuando el material refluido tiene un $\mathrm{pH}$ no ácido ${ }^{(5-7)}$.

La radiografía de esófago, estómago y duodeno (RxEED), fue uno de los primeros estudios utilizados para el diagnóstico de RGE, en la convicción de que con este examen era posible evaluar con certeza no sólo la presencia de este y su magnitud, sino también descartar una hernia hiatal, la que se consideraba estaba frecuentemente asociada $^{(1,8-9)}$. Estudios posteriores demostraron que su sensibilidad en la detección de RGE es baja, tanto en pacientes adultos como pediátricos, sin 
el uso de maniobras de provocación y no permite diferenciar entre reflujos fisiológicos y patológicos ${ }^{(10-}$ 12). Asimismo, se ha demostrado que el hallazgo de alteraciones anatómicas que expliquen los vómitos, es poco frecuente ${ }^{(13)}$ y que no existe diferencia significativa en la frecuencia de hernia hiatal entre pacientes con y sin RGE diagnosticado con $\mathrm{pH}$ metría (70\% y 63\% respectivamente) $)^{(13)}$. En nuestro medio, la RxEED es el método diagnóstico más utilizado para el diagnóstico de RGE en la actualidad.

Existen otros métodos diagnósticos que se han utilizado con menor frecuencia para evaluar el RGE, tales como la manometría, la cintigrafía, la endoscopía y la ultrasonografía(14-15).

Nuestra legislación permite otorgar licencia a aquellas madres cuyos hijos menores de un año presenten enfermedad grave ${ }^{(16)}$. Dentro de éstas, se incluye el RGE y el método diagnóstico utilizado para este objeto es la RxEED. Desde hace un tiempo, se ha planteado como un hecho, el uso ganancial de este examen para la obtención de los beneficios del sistema de salud por parte de las madres.

Por otro lado, desgraciadamente en nuestro país no está estandarizado el método de la RxEED, de tal manera que su resultado puede ser variable según quién sea el operador. Tampoco existe uniformidad en cuanto a su interpretación, lo que contribuye a aumentar más aún la confusión.

Los objetivos de este trabajo fueron: (a) evaluar la realidad que existe en el ambiente médico local, en cuanto a la utilidad e indicaciones de la RxEED, (b) tratar de determinar la proporción de exámenes que son solicitados sin haber una adecuada indicación médica, (c) buscar si existe o no evidencia que permita plantear el uso con fines gananciales de la RxEED en nuestro medio, por parte de las usuarias del sistema de salud y (d) intentar estandarizar la técnica de este método diagnóstico y uniformar su interpretación en nuestro país.

\section{Pacientes y método}

En forma prospectiva, se evaluó a todos los niños menores de 15 años, estudiados en forma consecutiva con RxEED en el Departamento de Radiología de nuestro Hospital Clínico, entre el $1^{\circ}$ de Abril y el 30 de Septiembre del

CASO No

\section{SINTOMAS}

Otros. año 2004. Se reunió un total de 190 pacientes, de los cuales 164 eran menores de un año, y en cada uno de ellos se consignó en forma sistemática, antecedentes en una ficha predeterminada, incluyendo información relativa a la técnica del examen, el cuadro clínico, el motivo del examen y un cuestionario a los padres (Anexo 1).

Técnica del examen: La técnica del examen fue la misma en todos los pacientes y corresponde a la utilizada desde hace muchos años en nuestro Departamento, basados en nuestra propia experiencia y en lo publicado en la literatura internacional ${ }^{(12-17)}$. Antes de comenzar el examen, se explicó el procedimiento a los padres y/o al niño, según el caso. Una vez instalado el paciente en la mesa de examen, se efectuó protección gonadal con dispositivo plomado. El medio de contraste utilizado fue sulfato de bario (HD200 plus, Lafayette Pharmaceuticals, Inc), en suspensión y a una concentración de $80 \%$, el que fue administrado por boca, a través de mamadera y/o

Anexo 1. Ficha usada para consignación de datos. Estudio RGE. Nombre Edad.

Fecha................Fono..... Médico tratante

Diagnóstico en solicitud de examen.

¿Reflejo de tos adecuado?

Infecciones respiratorias

Retardo ponderal.

Vómitos:..............duración... dolor abdominal.

Sospecha trastorno de la deglución

¿Esta en tratamiento por RGE? .......¿Con que tratamiento?...

¿Con respuesta clínica?.

¿Tiene RxEED anterior?..............Hallazgos.

TECNICA DEL EXAMEN.

Duración........... N $\mathrm{N}^{\circ}$ de exposiciones...........Volumen de bario

Edad gestacional..................... Peso nacimiento...

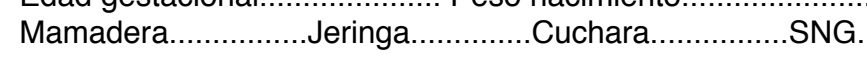

Tiempo de examen del RGE.................... ¿Llanto?..

Pasaje a vía aérea.................... Reflujo nasofaríngeo...

HALLAZGOS

Alteración de la deglución .....

Esófago:........................Normal.......

RGE: Espontáneo No........ Sí ........... N $\mathrm{N}^{\circ}$ de episodios

Cuantía: Abundante...

moderado leve.

Barrido esofágico

¿Significado patológico?

Vaciamiento gástrico

Ligamento de treitz normal..

.. Anormal.

PERCEPCIÓN DE LOS PADRES

¿Le explicó el pediatra el examen?.

¿Esta la madre o tutor con licencia por RGE del niño?

¿Cuánto tiempo?................................Isapre.

¿Tiene el examen relación con la suspención o extensión de la licencia?.......

¿Tiene otros exámenes?

¿Traumático para el niño? ................¿lo haría otra vez?

¿Deberían inventar un método más sencillo? 
jeringa y/o cuchara, según fuera necesario. El examen se inició con el paciente en decúbito lateral derecho, donde se obtuvo dos imágenes laterales incluyendo faringe, esófago y unión gastroesofágica. En este momento se evaluó dirigidamente los mecanismos de la deglución. Luego, con el paciente en decúbito supino, se obtuvo otras dos imágenes del esófago en proyección anteroposterior (AP). Posteriormente, se administró el resto del bario hasta obtener una adecuada distensión del fondo gástrico, para lograr así una oferta adecuada a la unión gastroesofágica, lo que se logró con una cantidad variable de contraste, según la edad del niño. En ningún caso se intentó llenar totalmente el estómago con contraste ni se persiguió alcanzar el volumen normalmente ingerido por el niño en cada alimentación. En ese momento y estando el paciente en decúbito supino e intentando mantener en contacto el bario con la unión esófago-gástrica se investigó RGE con fluoroscopia intermitente, con tiempos cortos de observación, por un período de aproximadamente 5 minutos completando en total un tiempo de radioscopia efectivo no mayor a 120 segundos, sin intentar ningún tipo de maniobras provocativas. En caso de observar RGE, se obtuvo una o dos imágenes adicionales y se constató el número de episodios durante el tiempo de observación, además de su cuantía, el tiempo de barrido esofágico y eventual pasaje a la vía aérea. Posteriormente, y con el paciente en decúbito lateral derecho, se evaluó el vaciamiento gástrico y el duodeno. La posición del ligamento de Treitz fue certificada con imágenes obtenidas en proyección lateral y AP. Finalmente, se obtuvo una imagen panorámica del estómago, duodeno y yeyuno proximal en AP.

\section{Resultados}

Del total de 190 pacientes, 104 eran niñas y 86 varones, con un promedio de edad de 9,7 meses y una mediana de 3 meses. El motivo del examen, en 157 fue un diagnóstico clínico presuntivo de RGE y el examen era solicitado para certificar este diagnóstico; de éstos, 100 (53 \%) no habían sido sometidos a ningún tipo de tratamiento médico para RGE. En este mismo grupo, 27 pacientes tenían un estudio radiológico previo donde se había demostrado RGE en 23 y en 4, este examen había sido reportado como normal. En 55 (28\%) pacientes el estudio se solicitó para evaluar respuesta al tratamiento médico. De los 190 pacientes, 33 fueron referidos con un diagnóstico diferente de RGE (Tabla I).

Cincuenta y cuatro pacientes (28\%) presentaban el antecedente de patología respiratoria. Ochenta y nueve madres (46\%) manifestaron que el examen era realizado para obtener una extensión o concesión de su licencia.

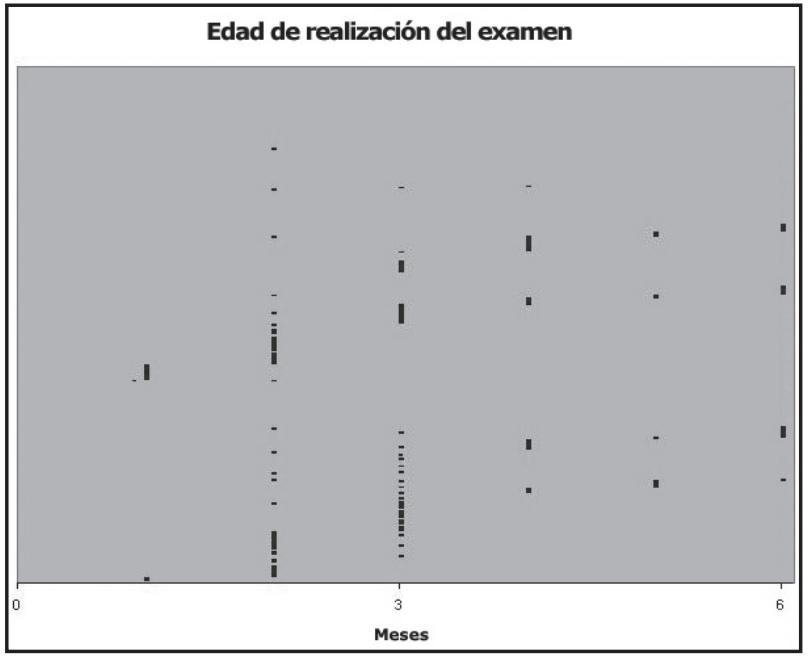

Gráfico 1. Distribución de los pacientes por edad (menores de 6 meses).

Tabla I. Diagnósticos clínico de referencia.

RGE 157

Bronquitis,tos, SBO

Hipersecrecion

Laringitis

Vómitos

Síndrome de Down

Trastorno de deglución

Anillo vascular

Chequeo

Cuadriparesia

Distensión abdominal

Dolor abdominal

Estenosis pilórica operada

Sin diagnóstico

Naúseas

Total

190

Al momento del examen, 164 niños (86\%) eran menores de un año y la mayoría de ellos se distribuyen entre los 2 y 3 meses (Gráfico 1). El promedio de edad en este grupo y cuyas madres no tenían licencia tipo IV, que corresponde a las extendidas a madres por enfermedad grave de niño menor de un año, fue de 3 meses, correspondiendo a 114 casos $(60 \%)$.

El volumen de bario promedio utilizado en menores de 6 meses fue $43 \mathrm{cc}$, entre 6 y 12 meses $58 \mathrm{cc}$; entre 13 y 36 meses $57 \mathrm{cc}$ y en mayores de 36 meses $75 \mathrm{cc}$.

El examen mostró RGE en un $46 \%$ de los pacientes y en $3(1,5 \%)$ se comprobó una alteración en la deglución. Sólo 14 niños de aquellos con diagnóstico clínico de RGE eran mayores de un año $(7,4 \%)$.

En ningún paciente se documentó anormalidad 
anatómica ni alteraciones evidentes del vaciamiento gástrico. En dos pacientes (1\%) se demostró ángulo de Treitz en línea media; pero en posición alta y posterior.

\section{Discusión}

El RGE es un fenómeno que, tanto niños como adultos, lo presentan de manera habitual, sin que ello necesariamente sea patológico ${ }^{(1,4,18)}$. Se ha considerado como normal un $\mathrm{pH}$ intraesofágico menor de 4 , hasta en un $5 \%$ del tiempo total de un día $(0.96$ $\mathrm{hr})^{(19-20)}$.

Se habla de enfermedad relacionada al RGE cuando éste produce síntomas, con o sin evidencias de esofagitis en la endoscopía(21) y el objetivo fundamental del tratamiento es aliviar estos síntomas, promoviendo el normal desarrollo, curar la esofagitis y evitar complicaciones respiratorias u otras.

La Academia Americana de Pediatría publicó recomendaciones en relación a los niños con RGE y enfermedades relacionadas y en ellas especifica que la historia clínica y el examen físico son suficientes para el manejo de esta condición, dejando los estudios por imágenes y de laboratorio para situaciones limitadas y específicas, como son la existencia de complicaciones relacionadas al RGE (retardo ponderal, patología pulmonar recurrente, etc.), la falta de resolución de síntomas de RGE a los 18 - 24 meses de vida, síntomas de alarma, retardo ponderal a pesar de adecuada oferta calórica, pirosis persistente a pesar de adecuado tratamiento, asma persistente a pesar de adecuado tratamiento, neumonías recurrentes, manteniéndose aún la controversia en relación a los episodios de $\operatorname{ALTE}^{(4)}$.

En la actualidad el examen de mayor certeza en el diagnóstico y detección de episodios de RGE ácido es la medición de pH intraesofágico en 24 horas, donde se registran los episodios de RGE medidos por el porcentaje de tiempo en que el $\mathrm{pH}$ es igual o menor de 4 , a través de una sonda fina y flexible colocada en esófago distal, considerando normal hasta un $5 \%$ del tiempo monitorizado ${ }^{(3)}$. De esta manera permite conocer no sólo el número de episodios de RGE en un período de 24 horas, sino también el tiempo de duración de cada uno de ellos y el tiempo total de pH ácido en el esófago. Sin embargo, no permite evaluar el volumen del material refluido y su rendimiento es menor cuando este tiene un $\mathrm{pH}$ no ácido (5-7). Los exámenes anormales pueden ser catalogados como RGE leve (6\%-9\%), moderado (10\%-19\%), o severo (más del $20 \%)^{(21)}$. Thomsom y cols. ${ }^{(22)}$, en una población de 117 pacientes que presentaban síntomas atribuidos a RGE, encontraron que solo un $60 \%$ de ellos reunía criterios diagnósticos de RGE, según $\mathrm{pH}^{(22)}$.

En nuestro medio, la RxEED es el método diagnóstico más ampliamente utilizado para el diagnóstico de RGE. Su sensibilidad ha sido reportada entre 26\% y 95\% (Tabla II), en la detección de RGE. Existe aún controversia en cuanto a la utilidad de la utilización de maniobras provocativas, como valsalva o el «sifonaje de agua», ya que algunos autores señalan un sustancial aumento de la sensibilidad en la detección del RGE ${ }^{(22)}$, pero otros establecen que esta mejoría en la sensibilidad es a expensas de una pérdida inaceptable de especificidad, por un aumento en los falsos positivos, tanto en pacientes pediátricos como adultos ${ }^{(12,19)}$. Los trabajos al respecto han comparado los hallazgos de la RxEED con los de la pH-metría de 24 horas, considerando esta última como gold standard. De esta manera, Chen y cols. ${ }^{(18)}$ reportaron que sólo un $30 \%$ de los pacientes que presentaba $\mathrm{pH}$-metría alterada, tenía signos radiológicos de enfermedad por RGE. Por otro lado, Thomson y cols. ${ }^{(22)}$ encontraron RGE espontáneo sólo en un $26 \%$ de aquellos pacientes con pH-metría alterada, pero al utilizar el «sifonaje de agua» y otras maniobras provocativas, este valor subía hasta $70 \%$. Sin embargo, estos autores no hacen mención del porcentaje de pacientes que tenían RGE, sin presentar pH-metría alterada.

Otro punto a evaluar, es la relevancia del volumen del material refluido. Thomson y cols. ${ }^{(22)}$ no encontraron una relación estadísticamente significativa entre el nivel alcanzado por el reflujo en el esófago, en la RxEED y el porcentaje del tiempo con $\mathrm{pH}$ menor de 4 en el estudio de $\mathrm{pH}$-metría, ni con los hallazgos en la endoscopía o la biopsia.

En cuanto al método utilizado para realizar el estudio radiológico, la mayor proporción del tiempo el paciente se encuentra en decúbito supino, lo cual ha sido demostrado aumenta los episodios de RGE en exámenes de monitoreo de $\mathrm{pH}$ en 24 horas ${ }^{(23-24)}$. El volumen de bario utilizado no ha sido estandarizado, pero la recomendación es utilizar una cantidad suficiente como para obtener una adecuada oferta a la unión gastroesofágica, evaluada bajo visión fluoroscópica. En nuestra experiencia, los volúmenes fueron proporcionales a la edad del niño, desde un promedio de $43 \mathrm{cc}$ en menores de 6 meses, hasta

Tabla II. Rendimiento de la RxEED en la detección de RGE.

\begin{tabular}{llcc} 
Autor & & Sensibilidad & Especificidad \\
\hline Blumhagen et al. & $(24)^{\star}+$ & $95 \%$ & $71 \%$ \\
Johnston et al. & $(13)^{*}$ & $26 \%$ & $77 \%$ \\
Aksglaede et al & $(12)^{\star}$ & $29 \%$ & $50 \%$ \\
Sellar et al. & $(25)$ & $71 \%$ & $75 \%$
\end{tabular}

Pacientes pediátricos, mayores y menores de un año. Con uso de maniobras de provocación. 
75 cc en mayores de 36 meses, valores similares a los reportados por Aksglaede y cols. ${ }^{(12)}$.

Desgraciadamente, en nuestro medio este examen no ha sido estandarizado, por lo que es muy difícil comparar la experiencia entre diferentes centros. Proponemos, estandarizar esta técnica, de acuerdo a lo antes mencionado, para lo cual sugerimos nuestra metódica como protocolo de examen.

Como ha sido demostrado la capacidad del examen para objetivar el RGE, es baja y mejora al utilizar maniobras provocativas, pero sin tener claro el significado de esto. Entonces, surge la inquietud respecto a qué le pedimos a la RxEED. En este sentido, la recomendación de la Academia Americana de Pediatría es utilizar este examen para la evaluación anatómica del tubo digestivo superior, en busca de patologías que puedan explicar la sintomatología de aquellos pacientes en que sea justificado descartar alteraciones tales como hernia hiatal, malrotación intestinal, etc. ${ }^{(4)}$.

Nuestros resultados son concordantes con lo expuesto en la literatura. De los 155 pacientes con diagnóstico clínico de RGE, sólo en 88 (46\%) se documentó su existencia durante el examen, destacando el hecho que no realizamos maniobras provocativas.

Como hemos destacado, la evidencia en la literatura es bastante clara con respecto a las indicaciones de la RxEED, las que son bastante acotadas, fundamentalmente dirigidas a la evaluación anatómica de un grupo seleccionado de pacientes. Sin embargo, en nuestros resultados comprobamos que solo $18(9 \%)$ de los pacientes presentaba una causa que podría justificar la indicación del examen, ya sea por persistencia de los síntomas sobre el primer año de vida o por signos de alarma asociados al RGE. Siendo así, nuestra recomendación es que el examen se concentre en búsqueda de alteraciones anatómicas, sin insistir en la demostración de RGE.

En nuestra legislación, según la ley 19.250, articulo $n^{\circ} 185$, se establece que: «Cuando la salud de un niño menor de un año requiera de atención en el hogar con motivo de enfermedad grave, circunstancia que deberá ser acreditada mediante certificado médico otorgado o ratificado por los Servicios que tengan a su cargo la atención médica de los menores, la madre trabajadora tendrá derecho al permiso y subsidio que establece el artículo anterior por el período que el respectivo Servicio determine. $\mathrm{Si}$ los beneficios precedentes fueren obtenidos en forma indebida, los trabajadores involucrados serán solidariamente responsables de la restitución de las prestaciones pecuniarias percibidas, sin perjuicio de las sanciones penales que por este hecho les pudiere corresponder»(15).

La licencia postnatal presenta una extensión de 84 días, es decir 2 meses y 24 días de vida del lactante. En nuestra experiencia y la de otros en nuestro medio ${ }^{(30)}$, el promedio de edad de los niños al momento de realizar el examen, fue de aproximadamente tres meses, coincidente con la finalización del período postnatal. Del total de las madres encuestadas, un 46\% manifestó directamente que realizaba el examen para la obtención de una licencia tipo IV.

Los datos obtenidos comprueban la creencia que existe un uso ganancial de la RxEED por parte de las usuarias del sistema de salud, aprovechando un vacío en la legislación que no define qué patologías deben ser consideradas graves, sin existir datos en la literatura que permitan afirmar que el RGE no complicado en niños menores de un año deba ser considerado patológico.

En este sentido, los radiólogos hemos sido partícipes de esto al hacer estos exámenes muchas veces innecesarios, con lo que hemos colaborado en la mantención de esta práctica en el tiempo. Surge además la responsabilidad ética de someter a un paciente a radiación innecesaria en un periodo de la vida en que sus potenciales riesgos son mayores.

Es evidente la necesidad de modificar la legislación, pues los datos en la actualidad son claros al respecto, y el gasto estimado es de al menos \$ 32 mil millones por este tipo de licencias, con un aumento en los últimos 11 años de $1.377 \%$ de gasto por concepto de este beneficio, de acuerdo a fuentes del Ministerio de Hacienda, lo que evidentemente repercute en un costo social que afecta a la hora del abordaje de verdaderas patologías.

\section{Resumen y conclusiones}

1) Nuestra experiencia muestra claramente el uso excesivo y muchas veces innecesario, de la RXEED en el estudio del niño con vómitos en nuestro medio. Médicos clínicos y radiólogos chilenos debemos uniformar criterios, basados en nuestra propia experiencia y en la internacional.

2) Es fundamental uniformar la técnica de la RxEED, para lo cual se propone un protocolo.

3) Es también necesario uniformar la manera de informar o reportar los resultados obtenidos, para facilitar a los médicos clínicos su interpretación.

4) Es imprescindible que nosotros radiólogos, conozcamos las indicaciones, limitaciones y rendimiento de la $R x$-EED.

5) Los fines gananciales de solicitud del examen por parte de los padres esta más allá de nosotros. Es evidente la necesidad de revisar la legislación al respecto, para que las madres trabajadoras puedan estar al lado de sus hijos cuando sea realmente necesario y sin tener que exponer a los niños a radiación innecesaria.

6) La Rx-EED no debiera ser un factor relevante al 
momento de decidir si otorgar o no licencia a la madre de un niño vomitador.

\section{Bibliografía}

1. Cosoi E. Reflujo Gastroesofágico en lactantes y niños. Pediatría al Día 1997; 13: 20-25.

2. Willich $\mathrm{E}$. The function of the cardias in childhood. J Pediatr Surg 1971; 3: 141-167.

3. Andze GO, Brandt ML, St Vil D et al. Diagnosis and treatment of gastroesophageal reflux in 500 children with respiratory symptoms: The value of $\mathrm{pH}$ monitoring. J Ped Surg 1991; 26: 295-300.

4. Pediatric GE Reflux. Clinical practice guidelines. J Pediatr Gastroenterol Nutr, 2001; 32, Suppl 2.

5. Dalt LD, Mazzolenni S, Montini G et al. Diagnostic Accuracy of $\mathrm{pH}$ monitoring in gastroesophageal reflux. Arch Dis Child 1989; 64: 1421-1426.

6. Johnson LF, De Meester TR. Twenty-four hour pH monitoring of the distal esophagus: a quantitative measurement of gastroesophageal reflux. Am J Gastroenterol 1974; 62: 325-332.

7. Mattox HE, Richter JE. Prolonged ambulatory esophageal $\mathrm{pH}$ monitoring in the evaluation of gastroesophageal Reflux Disease. Am J Med 1990; 89: 345-356.

8. Ott DJ, Glauser SJ, Ledbetter MS et al. Association of hiatal hernia and gastroesophageal reflux: Correlation between presence and size of hiatal hernia and 24 hour pH monitoring of the esophagus. AJR 1995; 165 : 557-559.

9. Mittai RK. Hiatal hernia and gastroesophageal reflux: Another attempt to resolve the controversy. Gastroenterology 1993; 105: 941-942.

10. Da Dalt L, Mazzoleni S, Riello D et al. Comparison of radiology and 24-hour esophageal $\mathrm{pH}$-monitoring in the diagnosis of gastroesophageal reflux in various pediatric age groups. Pediatr Med Chir. 1991; 13: 369-72.

11. Al-Khawari HA, Sinan TS, Seymour H. Diagnosis of gastro-oesophageal reflux in children. Comparison between oesophageal $\mathrm{pH}$ and barium examinations. Pediatr Radiol 2002; 32 :765-70. Epub 2002 Aug 09.

12. Aksglaede K, Pedersen JB, Lange A et al. Gastroesophageal reflux demonstrated by radiography in infants less than 1 year of age. Comparison with $\mathrm{pH}$ monitoring. Acta Radiol. 2003; 44: 136-8.

13. Johnston BT, Troshinsky MB, Castell JA, Castell DO. Comparison of barium radiology with esophageal $\mathrm{pH}$ monitoring in the diagnosis of gastroesophageal reflux disease. Am J Gastroenterol. 1996; 91:1181-5.

14. Gomes H, Menenteau B. Gastroesophageal reflux: Comparative study between sonography and $\mathrm{pH}$ monitoring. Pediatr Radiol. 1991, 21: 168-174.

15. Wrigth LL, Baker KR, Mery RG. Ultrasound diagnosis of gastroesophageal reflux. J Ultrasound Med 1988; 7: 471 .

16. Código del trabajo. DFL Número 1.- Santiago, 7 de Enero de 1994.- Subsecretaria del trabajo fija el texto refundido, coordinado y sistematizado del codigo del trabajo.

17. David A. Stringer. Pediatric Gastrointestinal imaging. 1989. B.C. Decker, Inc. Pags 19-20.

18. Guiraldes E. Larraín FJ, Harris P. Manual de Gastroenterología Pediátrica. Ediciones Universidad Católica de Chile. 1997. 115-132.

19. Rosen SN, Pope CE. Extended esophageal pH monitoring: an analysis of the literature and assessment of its role in the diagnosis and management of gastroesophageal reflux. J Clin Gastroenterol 1989; 11: 260-270.

20. Richter JE, Bradley LA, DeMeester TR, Wu WC. Normal 24 hour ambulatory esophageal $\mathrm{pH}$ values: influence of study center, $\mathrm{pH}$ electrodes, age and gender. Dig Dis Sci 1992; 37: 849-856.

21. Chen M, Ott D, Sinclari J, Wallace C. Gastroesophageal Reflux Disease: Correlation of $\mathrm{pH}$ testing and radiographic findings. Radiology 1992; 185: 483-486.

22. Thompson JK, Robert E, Koeh RE, Richter J. Detection of gastroesophageal reflux: Value of barium studies compared with $24 \mathrm{hrs} \mathrm{pH}$ monitoring. Am J Roengenl AJR 1994; 162: 621-626.

23. Byrne WJ, Euler AR, Campbell M. Body position and esophageal sphincter pressure in infants. Am J Dis Child. 1982; 136: 523-5.

24. Tutuian R, Elton JP, Castell DO et al. Effects of position on oesophageal function: Studies using combined manometry and multichannel intraluminal impedance. Neurogastroenterol Motil. 2003; 15: 63-7.

25. Ott DJ. Gastroesophageal Redlux: What is the role of barium studies? Am J Roentgenol AJR 1994; 162: 627-629.

26. Blumhagen JD, Christie DL. Gastresofageal reflux in children: Evaluation of water-siphon test. Radiology 1979; 131: 345-349.

27. Sellar Rj, De Caesttecker JS, Heading RC. Barium radiology: A sensitive test for gastroesophageal reflux. Clin Radiol 1987; 38: 303-307.

28. Guiraldes E. Pediatric Gastroenterology in Chile. A Personal Perspective. J Ped Gastroenterol 2001: 33: 231-232.

29. Meyers WF, Roberts CC, Johnson DG, Herbst JJ. Value of tests for evaluation of gastroesophageal reflux in children. J Pediatr Surg. 1985; 5: 515-20.

30. Arce JD. Comunicación personal. 